\title{
Outcomes of liver transplantation in hepatocellular carcinoma patients: a 10-year experience in a single tertiary center
}

\author{
Mati Rattanasakalwong, Methee Sutherasan
}

Department of Surgery, Chulalongkorn University, Bangkok, Thailand

Background: Liver transplantation is the gold standard treatment for patients with unresectable hepatocellular carcinoma (HCC), which are within Milan criteria. The recurrence rate after liver transplant was about $16 \%$ and the prognosis is poor. Predictors of recurrence after liver transplant have been widely studied, which are both morphologic features (tumor number and size), and biologic features (histopathology, tumor marker, and response to pretransplant treatment). This study aimed to report the outcomes of liver transplantation for $\mathrm{HCC}$ and determine the predictors of recurrence $\mathrm{HCC}$ after liver transplantation.

Methods: We performed a retrospective single-center of consecutive patients with HCC who underwent liver transplantation from 2009 to 2019. Demographic data, cause of HCC, tumor characteristics, and pathology of liver explants were collected. Primary outcomes were overall survival and disease-free survival. Predictors of recurrence were tested.

Results: Over a 10-year period, 105 patients underwent liver transplantation for HCC in our institution. The mean age was 57 years old. $73.3 \%$ (77/105) of patients were male. There were vascular invasions in $23.8 \%(25 / 105)$ of patients. Complete pathological response was found in $22.8 \%(24 / 105)$ of patients. $32.3 \%$ of patients were beyond Milan criteria. There were four recurrences (3.8\%) and 11 deaths (10.5\%) in this study. Median survival after recurrence was 11.5 months. Five-year OS and 5-year DFS were $88.2 \%$ and $89 \%$, respectively. Number of $\mathrm{HCC}$ ( $>3$ tumors) and vascular invasion were associated with recurrence odds ratio (OR) 17.2 (95\% confidence interval [Cl], 1.6-176.5; $\mathrm{P}=0.015)$ and $\mathrm{OR} 10.7(95 \% \mathrm{Cl}, 1.06-108.7 ; \mathrm{P}=0.041)$, respectively. In recurrence patients, there were more patients beyond Milan criteria and there was no complete pathological response but no statistical significance. No factor associated with overall survival was found.

Conclusions: In our data, the number of HCC more than three and vascular invasion were associated with HCC recurrence after liver transplantation.

Corresponding author: Methee Sutherasan

E-mail: matisurg@gmail.com

(c) The Korean Society for Transplantation

This is an Open Access article distributed under the terms of the Creative Commons Attribution Non-Commercial License (http://creativecommons.org/licenses/by-nc/4.0/) which permits unrestricted non-commercial use, distribution, and reproduction in any medium, provided the original work is properly cited. 